International Journal of Wireless \& Mobile Networks (IJWMN) Vol. 3, No. 5, October 2011

\title{
MULTI-PATH ROUTING AND CHANNEL ASSIGNMENT FRAMEWORK FOR MESH COGNITIVE RADIO NETWORK (MRCAMC)
}

\author{
${ }^{1}$ Amjad Ali, ${ }^{2}$ Muddesar Iqbal, ${ }^{1}$ Adeel Baig, ${ }^{3}$ Xingheng Wang \\ ${ }^{1}$ School of Electrical Engineering and Computer Sciences National University of \\ Science and Technology, Pakistan \\ $\{$ amjad.ali, adeel.baig\}@seecs.edu.pk \\ ${ }^{2}$ Faculty of Computer Science \& Information Technology, University of Gujrat, \\ Pakistan \\ m. iqbal@uog.edu.pk \\ ${ }^{3}$ College of Engineering, Swansea University, Swansea, UK \\ xingheng. wangeswansea. ac.uk
}

\begin{abstract}
Dynamic spectrum access is an attractive area of research these days. Cognitive Radio (CR) enabled networks are being deployed to effectively utilize the RF spectrum. Wireless mesh networks have been experiencing the bandwidth scarcity but such networks can easily enhance their throughput by using the $C R$ transceivers as these networks have the capability of multipath routing. Existing routing proposals for Mesh Cognitive Radio Networks (MCRNs) are not considering the dynamic spectrum availability. They try to treat CRNs as traditional wireless networks. Even some proposals treat the problems of these networks like that of wired networks and use the same parameter for route discovery as for wired networks. In this paper, we propose a joint interaction between on-demand routing and channel assignment that accounts the characteristics of CRNs.
\end{abstract}

\section{KEYWORDS}

CRNs, Routing, Mesh Cognitive Radio Networks

\section{INTRODUCTION}

Wireless communication has established itself as a popular access technology due to the user preference for flexibility, but its static channel allocation based spectrum management scheme is still a main problem. This static channel allocation scheme is very inefficient as it only allows the licensed users, Primary Users (PUs), to access the channels and does not permit unlicensed users, Secondary Users (SUs), to access the channels although the channels are idle or underutilized. This leads to the wastage of the spectrum resources. To efficiently utilize radio spectrum resources, a novel communication paradigm known as Cognitive Radio (CR) or Dynamic-Spectrum-Access (DSA) has been proposed[1] [2] [3]. Cognitive Radio Networks 
International Journal of Wireless \& Mobile Networks (IJWMN) Vol. 3, No. 5, October 2011

(CRNs) utilize the available spectrum opportunistically in the locality in which they operate. CR technology minimizes the wastage of radio spectrum band.

Wireless Mesh Networks (WMNs) [4] suffer from bandwidth scarcity. However, multipath routing can easily be achieved in WMNs. Thus splitting the flow among the multiple available paths can make simultaneous transmission to reduce the overall delay. Performance of WMNs can be elevated by using the CRs. Routing in Mesh Cognitive Radio Networks (MCRNs) [5] is a challenging task due to the diversity in the available channel set, data rates and reliability of the intermediate nodes. Without considering these issues routing cannot be useful in MCRNs as a large portion of useful time is utilized for routes discovery and route maintenance. Thus the critical issue in the MCRNs is the detection and avoidance of the PU interference as it affects the available channel set that further affects the route availability.

In MCRNs the existence of node itself has no significant meaning if the node has no common channel for communication with the neighboring nodes. If the node has some common channel with its neighbors for communications then we say node plays a key role in the network. Based on this argument we say the node/radio is virtual entity and channel/RF spectrum is physical entity. Thus deciding the route based on the physical location of the nodes/radios has no worth in MCRNs. Thus on demand routing technique is preferred for CRNs due to its dynamic nature. On demand protocol discovers the route from source to destination when it is required by source node instead maintaining the complete routing table for entire topology and updating it periodically. The mostly used on demand routing protocols are Dynamic Source Routing (DSR) [6] and Ad hoc on-demand Distance Vector (AODV) [7].

To the best of our knowledge, available routing proposals for the MCRNs select the intermediate nodes and channel on them heuristically without considering the dynamics of the CR. In this paper, we have proposed a new routing protocol for MCRNs that considers the dynamic of the CR. The rest of this paper is organized as follows. In Section 2 we discuss the related work and our motivation. In section 3 we present in detail the proposed routing protocol with network modeling. Paper conclusion and future work are discussed in section 4.

\section{RELATED WORK}

Research on the CRNs has started since last few years and researchers all over the world are exploring the new ways to deploy the CRNs such that the RF resources could be efficiently utilized. The routing proposals available for Mobile Ad hoc Networks (MANETs) and wireless mesh networks could not be directly applied for CRNs as they do not consider the dynamics of CRNs.

SMR [8] is on demand multipath routing protocol for MANETs. It discovers multiple disjoint paths between source and destination pair and splits the data traffic on multiple established paths. SMR selects the best route based on the minimum hop count. Furthermore it considers only single channel and node level disjointness while studies show that spectrum wise disjointness is more common in CRNs equipped with multiple interfaces [8].

Multi-Flow Real-Time Transport Protocol "MRTP" [9] is another multipath routing protocol for mesh-based MANETs. MRTP is based on the Real Time Protocol (RTP) and specifically used for multicast application. 
International Journal of Wireless \& Mobile Networks (IJWMN) Vol. 3, No. 5, October 2011

HC-IPSAG [10] is a cluster based routing proposal for CRN's. It splits the CRN's into clusters and each cluster is represented by head node. Each head node runs its own IPSAG protocol same like BGP.

Geographically based routing proposal for CRN's is proposed in [11], is derived from multihop, multi-channel adhoc and mesh networks. The proposed routing protocol focuses on most stable path that is indirectly achieved by simultaneous transmission over the multiple available channels and meeting the flow demand of CR node. It uses a novel routing metric based on probabilistic definition of available capacity over a channel.

In [12], a fuzzy based routing solution is proposed for CRN's. It enhances the throughput of CRN's by selecting the most stable channel when routes are being selected in addition to power factor that determines the amount of interference that primary user could afford. Channel stability is measured in term of channel utilization by primary user.

Multi-Radio Link-Quality Source Routing (MR-LQSR) [13] is multi-radio single path routing protocol for mesh-based MANETs. It uses link-state protocol for selecting the best path for source destination pair. Weighted cumulative expected transmission time (WCETT) is used for path selection. WCETT considers both link quality and minimum hop count.

SORP [14] and CARD [15] is single path routing protocols for MCRNs. Multipath Routing and Spectrum Access (MRSA) [16] is the only available multipath routing protocol for MCRNs. It is on demand and uses minimum hop count for selecting multiple paths between the source and destination pair. It handles the PU appearance on any path with the help of multiple paths used for same source destination pair. It does not consider the dynamics of CR while selecting the paths. Thus path selected by this routing protocol are not reliable while to ensure reliability is more important in CRNs than wired or other wireless networks.

\section{NETWORK MODEL}

Table 1: Index of symbols used in paper

\begin{tabular}{|c|c|}
\hline Symbol & Description \\
\hline$C_{n}(t)$ & Actual channel $\mathrm{n}$ status at time slot $\mathrm{t}$ \\
\hline$S_{n}(t)$ & Sensing result of channel $\mathrm{n}$ at time $\mathrm{t}$ \\
\hline$\delta_{n}, \varepsilon_{n}$ & Probability of errors in sensing results for channel $\mathrm{n}$ \\
\hline$\alpha_{n}, \beta_{n}$ & Transition probabilities of channel states \\
\hline$\mu_{n}(t)$ & Primary user susceptibility at time slot for channel $\mathrm{n}$ \\
\hline$p_{x}^{t r}$ & Transmission probability of node $x$ \\
\hline$\theta_{i}$ & Collision range \\
\hline$E_{S T X}$ & Successful transmission metric \\
\hline$E_{R A N K}^{x \leftarrow y}$ & Rank of a link between node y and node $\mathrm{x}$ \\
\hline $\mathfrak{N}$ & Set of Radios \\
\hline
\end{tabular}


International Journal of Wireless \& Mobile Networks (IJWMN) Vol. 3, No. 5, October 2011

\begin{tabular}{|c|c|}
\hline$\mu_{i, c_{l}}$ & Primary user susceptibility on link $l$ for channel of path $\mathrm{i}$ \\
\hline $\mathfrak{c}_{k, r}$ & Candidate channel of node $k$ for radio $r$ \\
\hline${ }^{\circ} \mathrm{C}_{k, j}$ & Common channels between node $k$ and $\mathrm{j}$ \\
\hline $\mathbb{R}_{\boldsymbol{i}}$ & Rank of path $i$ \\
\hline
\end{tabular}

\subsection{Primary Users Susceptibility}

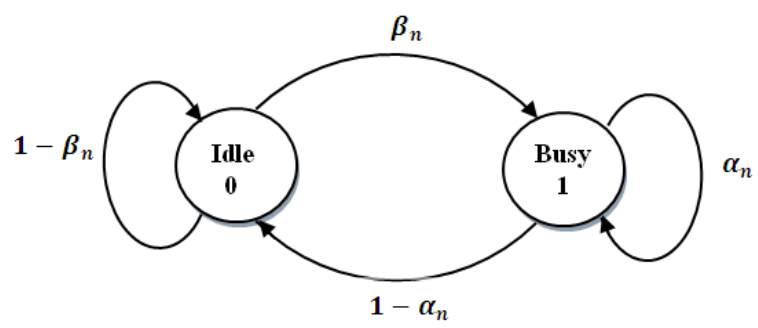

Figure1. Markov model for channel status

Let $\mathrm{N}=1,1,(1,2, \ldots, \mathrm{n})$ channels available in the whole spectrum band ${ }^{\Psi}$. We assume that an individual node is only capable of accessing and sensing a subset of the whole spectrum, i.e. $\beta_{\mathrm{i}} \subseteq \Psi$. let the channel availability for the secondary user be modeled by a Markov process given in Fig 1. To cater for the errors in the sensing process we can formulate two vectors, one consisting of the actual condition the channel is in and the other being the actual results of the sensing process. $\mathrm{S}_{n}(\mathrm{t})$ denotes the sensing results achieved by a node and let $\mathrm{C}_{n}(t)$ denote the actual channel state the channel was in. Since the channel can be in either of the two states, i.e. busy or idle, the errors induced in the sensing process and resulting in a false value of $S_{n}(t)$ can be represented by asymmetrical channel depicted in Fig. 2.

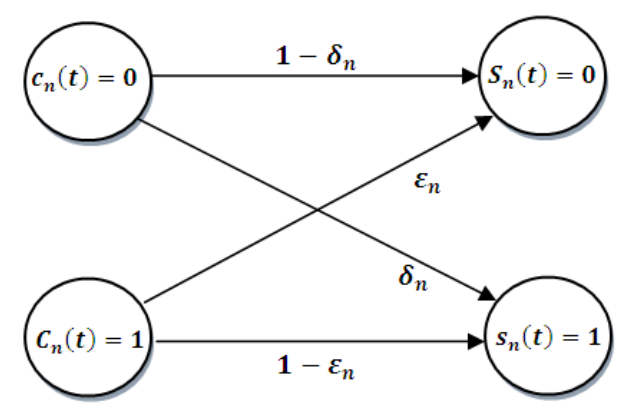

Figure2. Asymmetric channel for actual channel status and sensing results of node

The errors in the sensing process are defined by the following equations: 


$$
\begin{aligned}
& \operatorname{Prob}\left(\mathrm{S}_{\mathrm{n}}(\mathrm{t})=1 \mid \mathrm{C}_{\mathrm{n}}(\mathrm{t})=0\right)=\delta_{\mathrm{n}} \\
& \operatorname{Prob}\left(\mathrm{S}_{\mathrm{n}}(\mathrm{t})=0 \mid \mathrm{C}_{\mathrm{n}}(\mathrm{t})=1\right)=\varepsilon_{\mathrm{n}}
\end{aligned}
$$

The sensing results and the actual channel status are defined by a vector. The sensing vector is given by $\overrightarrow{\mathrm{S}_{\mathrm{n}}}(\mathrm{t})=\left[\mathrm{S}_{1}(\mathrm{t}), \mathrm{S}_{2}(\mathrm{t}), \ldots, \mathrm{S}_{\mathrm{n}}(\mathrm{t})\right]$ and the actual channel condition vector is given by $\overrightarrow{C_{n}}(t)=\left[C_{1}(t), C_{2}(t), \ldots, C_{n}(t)\right]$. We define primary user susceptibility for a channel as a belief of the channel being in busy state. The primary user susceptibility vector would then be given by $\overrightarrow{\mu_{\mathrm{n}}}(\mathrm{t})=\left[\mu_{1}(\mathrm{t}), \mu_{2}(\mathrm{t}), \ldots, \mu_{\mathrm{n}}(\mathrm{t})\right]$. Hence by utilizing Bayes law and conditional probability we can compute $\mu_{\mathrm{n}}$ in the following way. The probability of channel being in the busy state, given some previous history of the channel $\omega_{n}$ is given by

$$
\begin{aligned}
& \mu_{n}(t)=\operatorname{Prob}\left(C_{n}(t)=1 \mid \omega_{n}\right) \\
= & \alpha_{n} \mu_{n}(t-1)+\beta_{n}\left(1-\mu_{n}(t-1)\right) \\
= & \pi_{n}(t)
\end{aligned}
$$

Equation (3) defines the primary user susceptibility as the channel being in the busy state in the previous time slot or having moved in the busy state from the idle state in the previous time slot. The history $\omega_{\mathrm{n}}$ can be manipulated as:

1. If there is an acknowledgement received in time interval $t$, then in time $t-1$, the channel was busy in data transmission.

2. If there is a successful negotiation for transmission in the current slot then in the previous slot, the channel was busy in carrying requests.

3. If there is data received in time interval $t$ then in $t-1$, the channel was busy in transmission.

Incorporating and conditioning on the channel sensing result $S_{n}$, we can write for correctly deciding upon the available channels as

$$
\begin{gathered}
\mu_{n}(t)=\operatorname{Prob}\left(C_{n}(t)=1 \mid S_{n}(t)=1, \omega_{n}\right) \\
\mu_{n}(t)=\frac{\operatorname{Prob}\left(S_{n}(t)=1 \mid C_{n}(t)=1, \omega_{n}\right)}{\sum_{I_{n} \epsilon(0,1)} \operatorname{Prob}\left(S_{n}(t)=1 \mid C_{n}(t)=I_{n}, \omega_{n}\right)} \\
=\frac{\operatorname{Prob}\left(S_{n}(t)=1 \mid C_{n}(t)=1\right) \operatorname{Prob}\left(C_{n}(t)=1 \mid \omega_{n}\right)}{\sum_{I_{n}} \operatorname{Prob}\left(S_{n}(t)=1 \mid C_{n}(t)=I_{n}\right) \operatorname{Prob}\left(C_{n}(t)=I_{n} \mid \omega_{n}\right)} \\
\mu_{n}(t)=\frac{\pi_{n}(t)\left(1-\varepsilon_{n}\right)}{\pi_{n}(t)\left(1-\varepsilon_{n}\right)+\left[1-\pi_{n}(t)\right] \delta_{n}}
\end{gathered}
$$


Where the value of $\pi_{n}(t)$ will be replaced as $\alpha_{n} \mu_{n}(t-1)+\beta_{n}\left(1-\mu_{n}(t-1)\right)$. Values of $\varepsilon_{n}$ and $\delta_{\mathrm{n}}$ can be computed by running the system on some time scale [0-T] and updated after a significant percentage change $\Delta$ occurs in the value, hence it is specific to a particular nodes operation. We have defined the probability $\mu_{n}(t)=\pi_{n}(t)$ as a function of previous time interval. Hence by performing a recursive operation we can write for any future time instant $t+$ $\tau$, the probability value as

$$
\mu_{\mathrm{n}}(\mathrm{t}+\tau)=\mu_{\mathrm{n}}(\mathrm{t})\left(\alpha_{\mathrm{n}}-\beta_{\mathrm{n}}\right)+\beta_{\mathrm{n}} \sum_{\mathrm{i}=0}^{\tau-1}\left(\alpha_{\mathrm{n}}-\beta_{\mathrm{n}}\right)^{\mathrm{i}} \forall \tau>0
$$

The $\mu_{n}(t+\tau)$ value is used in algorithm 2 for computing the channel status over a period of time. Hence if a node is unable to perform channel sensing at a particular slot, it can use the approximations from this equation.

Here we try to formulate a metric that can combine the effect of links on a particular hop while also providing information of the total hops traversed. A metric that gives proportional importance to the combined channel states will suit the purpose. We have already defined the probability with which a node is sure that a channel will not be susceptible to the primary user or simple the probability $\mu_{n}(t)$ with which the channel would be idle. We can define here the probability for unsuccessful transmission $\mathrm{p}$ between a node $\mathrm{x}$ and $\mathrm{y}$ in the form of busy channel and transmission probability. Let the transmission probability be given by $\mathrm{p}_{\mathrm{x}}^{\mathrm{tr}}$ for node $\mathrm{x}$ and $\mathrm{p}_{\mathrm{y}}^{\mathrm{tr}}$ for node $\mathrm{y}$. Then for unsuccessful transmission on a bidirectional link basis $\mathrm{p}$ can be defined as

$$
\mathrm{p}=1-\left[1-\left(\mu_{\mathrm{n}}^{\mathrm{x}}(\mathrm{t})\right)\left(\mathrm{p}_{\mathrm{x}}^{\mathrm{tr}}\right)\right] \times\left[1-\left(\mu_{\mathrm{n}}^{\mathrm{y}}(\mathrm{t})\right)\left(\mathrm{p}_{\mathrm{y}}^{\mathrm{tr}}\right)\right]
$$

Where $\mu_{\mathrm{n}}^{\mathrm{x}}(\mathrm{t})$ and $\mu_{\mathrm{n}}^{\mathrm{y}}(\mathrm{t})$ are the probabilities of miss detection, i.e. the channel was in busy state and the sensing result was a 0 (busy). Similar to equation (4), we can compute the probability of miss detection as

$$
\begin{array}{r}
\mu_{n}^{x, y}(t)=\operatorname{Prob}\left(C_{n}(t)=1 \mid S_{n}(t)=0, \omega_{n}\right) \\
\mu_{n}^{x, y}(t)=\frac{\pi_{n}(t)\left(\varepsilon_{n}\right)}{\pi_{n}(t)\left(\varepsilon_{n}\right)+\left[1-\pi_{n}(t)\right]\left(1-\delta_{n}\right)}
\end{array}
$$

The probability of transmission can be defined in terms of a collision range under which we allow a node to transmit. Let this range be termed as $\theta_{\mathrm{i}} \in(0,1)$. As we want the collision to be as limited as it can be, we set

$$
p_{i}^{\operatorname{tr}}(t)\left(\mu_{n}^{x, y}(t)\right) \leq \theta_{i}
$$


International Journal of Wireless \& Mobile Networks (IJWMN) Vol. 3, No. 5, October 2011

That is a node senses the channel falsely to be idle and transmits causing a collision should be limited by $\theta_{\mathrm{i}}$. Therefore a node transmits with the following probability.

$$
\mathrm{p}_{\mathrm{i}}^{\mathrm{tr}}(\mathrm{t})=\min \left(1, \frac{\theta_{\mathrm{i}}}{\mu_{\mathrm{n}}^{\mathrm{x}, \mathrm{y}}(\mathrm{t})}\right)
$$

Equation (9) shows the transmission being done in opportunistic or a deterministic way. If the value $\frac{\theta_{i}}{\mu_{n}^{X, y}(t)}$ is larger than 1 , then the node will deterministically transmit with a probability of 1 , otherwise opportunistically with a value of $\frac{\theta_{i}}{\mu_{n}^{x, y}(t)}$. Assuming the successful and unsuccessful attempts of packet transmission as Bernoulli trials, we can write the successful transmission probability after $\mathrm{k}$ attempts as

$$
\mathrm{P}_{\text {suc }}=\mathrm{p}^{\mathrm{k}-1} \times(1-\mathrm{p})
$$

Hence finally we can write a metric that incorporate the total number of transmission attempts required to successfully transmit a packet from node $\mathrm{x}$ to node $\mathrm{y}$. we call this metric as $\mathrm{E}_{\mathrm{STX}}$ and it inherently incorporates the link layer failures at a particular node.

$$
\mathrm{E}_{\text {STX }}=\sum_{\mathrm{k}=1}^{\propto} \mathrm{k} \times \mathrm{P}_{\text {suc }}
$$

The value of $E_{\mathrm{STX}}$ describes the characteristics achieved on a single link for a particular channel. However there can be multiple channels that are common between two nodes. So we define another metric that depicts the combined effect of all the common channels on a link between two nodes xand $y$. Let

\section{$k \in$ set of working channels on node $\mathrm{x}$ \\ $l \in$ set of working channels on node y}

Therefore, we are interested in the channels that are common between the communicating nodes $\mathrm{x}$ and $\mathrm{y}$ and define the variable $\mathrm{i}$ as

$$
\mathrm{i}:(\mathrm{i} \subseteq \Psi) \text { and }(\mathrm{i} \in(\mathrm{k} \cap \mathrm{l}))
$$

Therefore the combined effect over the common channels is defined as

$$
\mathrm{E}_{\mathrm{RANK}}^{\mathrm{x} \leftarrow \mathrm{y}}=\sum_{\mathrm{i}} \mathrm{E}_{\mathrm{STX}}
$$

The metric $\mathrm{E}_{\mathrm{RANK}}^{\mathrm{xy}}$ has the property of bi-directionality and. We call this as the rank given by a node to in the backward direction neighbor. 
International Journal of Wireless \& Mobile Networks (IJWMN) Vol. 3, No. 5, October 2011

\section{Algorithm 1: Instantaneous Rank Calculation}

1: Input [Neighbour y, Channel List L]

2: Compute: $\mathrm{j}:(\mathrm{j} \subseteq \Psi)$ and $(\mathrm{j} \in(\mathrm{k} \cap \mathrm{l}))$

3: Index the list with $\mathrm{j}$

4: For (1 to $\mathrm{j})$

5: Compute: $\mu_{\mathrm{n}}(\mathrm{t}) \leftarrow\left(\varepsilon_{\mathrm{n}}, \delta_{\mathrm{n}}\right)$

6: Compute: $\mathrm{P} \leftarrow \mu_{\mathrm{n}}(\mathrm{t}), \mathrm{p}_{\mathrm{n}}^{\mathrm{tr}}$

7: Compute: $\mathrm{P}_{\text {suc }} \leftarrow \mathrm{p}, \mathrm{k}$

9: Compute $\mathrm{E}_{\text {STX }} \leftarrow \mathrm{P}_{\text {suc }}, \mathrm{k}$

10: Update $\mathrm{E}_{\mathrm{RANK}}$

11: End for

Algorithm 1 computes the $\mathrm{E}_{\mathrm{RANK}}$ value for a specific neighbor when a request route discovery request comes. The algorithm computes the common data channels between the two nodes and then computes the probability of success on these channels in an iterative manner. Finally an up to date value of the $E_{\text {RANK }}$ value is used for ranking the link with that node

\section{Algorithm 2: Periodic Rank Calculation}

1: Input [Neighborset $\mathrm{N}$, Channel List L]

2: Initialize Rank $=[\mathrm{N}]$

3: Index nodes with $\mathrm{i}$

4: For (1 to i)

5: Compute: $\mathrm{j}:(\mathrm{j} \subseteq \Psi)$ and $(\mathrm{j} \in(\mathrm{k} \cap \mathrm{l}))$

6: Index the list with $\mathrm{j}$

7: For ( 1 to $\mathrm{j})$

8: Compute: $\mu_{\mathrm{n}}(\mathrm{t}) \leftarrow\left(\varepsilon_{\mathrm{n}}, \delta_{\mathrm{n}}\right)$

9: Compute: $\mathrm{P} \leftarrow \mu_{\mathrm{n}}(\mathrm{t}), \mathrm{p}_{\mathrm{n}}^{\mathrm{tr}}$

10: Compute: $\mathrm{P}_{\text {suc }} \leftarrow \mathrm{p}, \mathrm{k}$

11: Compute $\mathrm{E}_{\text {STX }} \leftarrow \mathrm{P}_{\text {suc }}, \mathrm{k}$

12: Update $\mathrm{E}_{\mathrm{RANK}}$

13: End for

14: $\operatorname{Rank}[\mathrm{i}]=\mathrm{E}_{\mathrm{RANK}}$

15: End for 
International Journal of Wireless \& Mobile Networks (IJWMN) Vol. 3, No. 5, October 2011

Algorithm 2 computes the ranks of the complete neighbor set of a node and used for ranking the links in a periodic manner. Equation (5) is used in the algorithm for computing the $\mu_{n}(t)$ values between the time interval of the period and a vector set is maintained. These results can be used by any node which is unable to perform sensing in a particular time interval.

\subsection{Assumptions}

First, we assume that each network node contains 3 radios for data traffic. Secondly all signaling/routing information uses a common control channel that is available every time and tuned on a separate dedicated radio. Thus there are total $3+1$ radios at each node. Thirdly, we assume that the link existing between two communicating node is bi-directional. Lastly, we assume that at least $40 \%$ of the secondary user communication should be succeeded on every selected link in path. Therefore, we define a threshold $E_{\text {LIMIT }}$ equal to 0.4. Also the path establishment and channel assignment is being done for a single existing source destination pair in the network.

\subsection{Route Discovery}

In the routing module of our proposed framework, we use the on-demand routing mechanism to build the multiple routes for a single source destination pair. Thus we modify Dynamic Source Routing (DSR) protocol for this purpose. When source node wants to communicate with destination node and no route information is known, source node initiates ROUTE REQUEST (RREQ) with new ID and broadcasts it to its 1-Hop neighbors. This process continues till the destination node receives multiple RREQ from different routes. Then the destination node assigns channel and radio to each link of the candidate path according to Algorithm 3. After this effectiveness of each candidate path is evaluated so destination can select a maximum of 4 best routes for the source. Maximum three of them will be used for the data communication and the other path will be preserved and used as a backup route, to be discussed in detail later. Selected route information is sent to the source node via the ROUTE REPLY (RREP) packet that is unicast. The selected intermediate nodes will tune their radio and channel accordingly.

\subsection{RREQ Propagation}

The main objective of our routing module is to construct maximally disjoint paths. Two types of disjointness; node level and spectrum level will be ensuring to construct the multiple routes. This is to prevent some nodes being congested and to efficiently utilize the network resources. Therefore it is necessary that the destination node knows the information of all the candidate paths to ensure disjointness. Thus we use the source routing approach where each forwarding node including source append their information with the RREQ message. Furthermore intermediates nodes are not allowed to send RREP to source in case they have route information in their cache.

When the source node has some data for destination node but does not have path information. It will generate a new RREQ packet with new RREQ_ID that uniquely identify the RREQ packet. Source node appends its ID and Radio, Channel, Susceptibility (RCS) table. When an intermediate node receives RREQ it will firstly ensure that the RREQ is not duplicate by checking its ID in appending list if so then discards this RREQ. If not then appends its node ID and RCS table and forwards it. In order to discover multiple paths between source and 
International Journal of Wireless \& Mobile Networks (IJWMN) Vol. 3, No. 5, October 2011

destination pair we introduce a new forwarding approach based on our metric $\mathrm{E}_{\mathrm{RANK}}^{\mathrm{x} \leftarrow \mathrm{y}}$. Thus each intermediate node processes the RREQ as follow:

1. Check whether there exists some common data channel with the forwarded node if no then discard the RREQ.

2. Compute the $\mathrm{E}_{\mathrm{RANK}}^{\mathrm{X} \leftarrow \mathrm{y}}$ according to Algorithm 1 and compare with the $\mathrm{E}_{\text {LIMIT }}$. If the $\mathrm{E}_{\mathrm{RANK}}^{\mathrm{X} \leftarrow \mathrm{y}}$ value is below the threshold then discard RREQ packet, forward otherwise.

3. If a RREQ request packet from the same ID was processed earlier from a different forwarded neighbor, then it will compute $\mathrm{E}_{\mathrm{RANK}}$ vector according to the Algorithm 2 and compare the corresponding column entries. For a greater value of $\mathrm{E}_{\mathrm{RANK}}^{\mathrm{X} \leftarrow \mathrm{y}}$ for the current request as compared to the previously processed RREQ packet, the packet will be forwarded, discarded otherwise.

4. If the $E_{\text {RANK }}$ value for both RREQ packets will be the same then Hop count will be used as tie breaker.

5. To avoid loops the packet is discarded by the previous node from where the packet had been forwarded when received.

6. Every intermediate node in the path appends its ID and Band, Radio, Channel Susceptibility (BRCS) table. The format of the table is as follows:

Table 2: The BRCS Table

\begin{tabular}{|c|c|c|c|}
\hline \multicolumn{4}{|c|}{ Node ID } \\
\hline $\begin{array}{c}\text { Band } \\
\text { B }\end{array}$ & $\begin{array}{c}\text { Susceptib } \\
\text { ility } \\
\text { S }\end{array}$ & $\begin{array}{c}\text { Neighb } \\
\text { ors } \\
\mathbf{N}\end{array}$ & $\begin{array}{c}\text { Rad } \\
\text { io } \\
\mathbf{R}\end{array}$ \\
\hline$b_{1}$ & $\mu_{1}$ & \multirow{3}{*}{$n_{1}, n_{2}$} & \multirow{3}{*}{$r_{1}, r_{2}, l$} \\
\hline$b_{2}$ & $\mu_{2}$ & & \\
\hline$b_{3}$ & $\mu_{3}$ & & \\
\hline
\end{tabular}

The table includes the nodes ID, the data channels available at the node. The destination node waits a certain period of time to collect more RREQ from different paths and apply Algorithm 3 to assign channel and radio on each path. Note that all the routes are not necessarily of equal quality and length.

Hence if a request had traversed from source node $\mathrm{S}$ to destination node $\mathrm{D}$ through hops $\mathrm{A}, \mathrm{B}$ and $\mathrm{C}$, following IDs would have been appended in the described sequence when the request reaches node $\mathrm{D}$.

Table 3: The PREQ format

\begin{tabular}{|l|l|l|l|}
\hline Node S & Node A & Node B & Node C \\
\hline
\end{tabular}


International Journal of Wireless \& Mobile Networks (IJWMN) Vol. 3, No. 5, October 2011

\subsection{Path Selection and Channel Assignment}

It selects a channel for each link along the path by considering the two factors: firstly channel is not already assigned within one hope neighbor, secondly channel with minimum susceptibility among the common channel set of link should be selected. The destination waits a certain amount of time to gather multiple PREQ's from different paths. The destination evaluates each path by choosing the channel on each link with minimum susceptibility and avoiding the selection of same channel in the interference range neighbors. Algorithm followed by destination for path selection is described in Algorithm 3.

\section{Algorithm 3: Path Selection Algorithm}

1: Input: [RREQ (m)]

2: for $i: 1$ tom

3: for $j=\mathrm{n}$ to 2 where $\mathrm{n}$ is number of nodes in path

4: $\mathrm{k}=j-1$

5: ${ }^{\circ} \mathrm{C}_{\mathrm{k}, \mathrm{j}}=(\operatorname{channels}(j) \cap \operatorname{channels}(k))$

6: $\quad c=\arg \min c \in{ }^{\circ} \mathrm{C}_{\mathrm{k}, \mathrm{j}}\left(\mu_{\mathrm{l}}\right) \quad \mathrm{l} \in{ }^{\circ} \mathrm{C}_{\mathrm{k}}$

7: while $c \in \mathfrak{c}_{p} \cup \mathfrak{c}_{k}$ where $\mathrm{p} \in$ Neighbours of $\mathrm{k}$,

8: $c_{p}$ is candidate channel for $\mathrm{p}$

9: ${ }^{\circ} \mathrm{C}_{\mathrm{k}, \mathrm{j}}={ }^{\circ} \mathrm{C}_{\mathrm{k}, \mathrm{j}} \backslash \mathrm{c}$

10: $\mathrm{c}=\arg \min \mathrm{c} \in{ }^{\circ} \mathrm{C}_{\mathrm{k}, \mathrm{j}}\left(\mu_{\mathrm{l}}\right) \quad \mathrm{l} \in{ }^{\circ} \mathrm{C}_{\mathrm{k}, \mathrm{j}}$

11: end while

12: $\mathfrak{c}_{\mathrm{k}, \mathrm{r}}=\mathfrak{c}_{\mathrm{k}, \mathrm{r}} \cup \mathrm{c} \quad \mathrm{r} \in \mathfrak{N} \quad \mathrm{r}$ is less expected used radio

13: end for

14: $\quad \mathbb{R}_{\mathrm{i}}=\sum_{\mathrm{k}=2}^{\mathrm{n}} \mu_{\mathrm{i}, \mathrm{c}_{\mathrm{k}}} /$ no. of hops

15: end for

After receiving sufficient number of RREQ with same RREQ ID from different path, destination node computes the candidate channel for each link along the path. Candidate channel is the common channel between two nodes $k$ and $j$ with minimum $\mu_{n}$ and also it should not be in candidate channel set of neighbors of node $k$ as well as in its own candidate channel set. If channel with minimum $\mu_{\mathrm{n}}$ is also in candidate channel set of neighbors of node $k$ or in its own candidate channel set then remove channel from the common channel list of $\mathrm{k}$ and $j$. Again compute the channel with minimum $\mu_{\mathrm{n}}$ until it satisfies both conditions. Following same procedure, destination determines the candidate channel for each node along the path. In order to get primary user susceptibility for a path, sum of susceptibility on candidate channel of each link along the path is divided by number of hops. Three paths among the discovered paths with 
International Journal of Wireless \& Mobile Networks (IJWMN) Vol. 3, No. 5, October 2011

minimum rank are used in multipath routing and another can be set as a backup path. At the end of the procedure the destination node sends a ROUTE REPLY (RREP) message along the chosen paths to set the channels on the links.

\section{Conclusion}

The problem that the paper focuses is to discover multiple routes in cognitive mesh networks using a different methodology than previously adopted hop count and then do channel assignment on the nodes. We have defined a probability measure as primary user susceptibility on a channel sensed by a node as the percentage of the channel being in busy state. This has been done by defining a combination of markov and asymmetric channel models. This incorporates the basic characteristics of cognitive radios. From it we used a communication success probability on a channel and used this to rank the link and define a metric that can be used to forward or drop route establishment requests in terms of a defined threshold. Hence here if channels on a link are having high success rates, the requests would be forwarded and multiple paths would be established even if we are having more hops. The request contains appended data from intermediate hops in the form of channels and the primary user susceptibility. The destination then selects path preferences by the combined effect of primary user susceptibilities on the channels in the path and avoiding inter and intra flow interferences at the same time while assigning channels to the nodes.

\section{ACKNOWLEDGEMENTS}

I would like to special thanks for Miss Saba Saifullah for making available the data and other help. This work could not be completed without her help.

\section{REFEREENCES}

[1] Joseph Mitola III ANd Gerald Q. MaguiRe. Cognitive Radio:MaKing SOFTWARE RAdios MORE PERSONAL. IEEE PERSONAL COMMUNICATIONS, 6:13-18, 1999.

[2] Simon Haykin. Cognitive radio: Brain-empowered wireless communications. IEEE Journal Selected Areas in Communications, 23:201-220, 2005.

[3] Ian F Akyildiz, Won Yeol Lee, Mehmet C Vuran, and Shantidev Mohanty. Cnext generation/dynamic spectrum access/cognitive radio wireless networks: A survey. Computer Networks, 50:2127-2159, 2006.

[4] IEEE 802.11s http://www.802wirelessworld.com/.

[5] R. Hincapie, J. Tang, G. Xue and R. Bustamante, QoS routing in wireless mesh networks with cognitive radios, Proceedings of IEEE Globecom'2008.

[6] D.B. Johnson and D.A. Maltz, .Dynamic Source Routing in Ad Hoc Wireless Networks, In Mobile Computing, 1996.

[7] C.E. Perkins and E.M. Royer, .Ad-Hoc On Demand Distance Vector Routing, Proceedings of IEEE WMCSA'99, 1999.

[8] Sung-Ju Lee and Mario Gerla. Split multipath routing with maximally disjoint paths in ad hoc networks. In Proceedings of IEEE ICC, 2001. 
International Journal of Wireless \& Mobile Networks (IJWMN) Vol. 3, No. 5, October 2011

[9] Shiwen Mao, Dennis Bushmitch, Sathya Narayanan, and Shivendra S. Panwar, "MRTP: A MultiFlow Realtime Transport Protocol for Ad Hoc Networks", Vehicular Technology Conference, 2003.

[10] Badoi, C.-I, Croitoru, V, Popescu, A. "HC-IPSAG Cognitive Radio Routing Protocol: Models and Performance" in 8th International Conference on Wireless and Optical Communications Networks (WOCN), 2011.

[11] H.Khalife, S.Ahuja, N.Malouch and M.Krunz. "Probabilistic Path Selection in Opportunistic Cognitive Radio Networks" In the proceedings of the IEEE globecom conference. Orleans, USA 2008.

[12] A. El Masri, N. Malouch. "A Routing Strategy for Cognitive Radio Networks Using Fuzzy Logic Decisions", the First International Conference on Advances in Cognitive Radio: COCORA 2011.

[13] R. Draves, J. Padhye, and B. Zill, "Routing in Multi-Radio, Multi-Hop Wireless Mesh Networks," ACM Annual Int'l. Conf. Mobile Comp. and Net. (MOBICOM), 2004.

[14] G. Cheng, W. Liu, Y.Z. Li, and W.Q. Cheng. Spectrum aware on-demand routing in cognitive radio networks. In Proceedings of IEEE DySPAN, 2007.

[15] G. Chittabrata and A. Dharma P. Channel assignment with route discovery (card) using cognitive radio in multi-channel multi-radio wireless mesh networks. In Proceedings of $1^{\text {st }}$ IEEE SDR Workshop, IEEE SECON, 2006.

[16] Wang, X. and Kwon, T.T. and Choi," A multipath routing and spectrum access (MRSA) framework for cognitive radio systems in multi-radio mesh networks" Proceedings of the 2009 ACM workshop on Cognitive radio networks. 